\title{
Editorial
}

\section{Potency, Action, Standardization, and Debate - The High Dilution Way}

Saurav Arora

The latest issue of International Journal of High Dilution Research features articles on long debated issues such as potency estimation, mode of action of homeopathic drugs, standardization, homeoprophylaxis and dosage.

In addition to the absence of "original drug molecules" in ultra-high dilutions (UHD), an equal challenge is the estimation of potency. The potentization process is controversial from physicochemical, mechanical as well as philosophical point of view. As UHD surpass Avogadro's constant, therefore, it is believed that the potency estimation is practically impossible. To address this vital issue Nandy et al propose a mathematical formula based upon experiments conducted by them, where they relate the size of nanoparticles (NPs) with potency. The authors propose that size of NPs decrease with potency, with which it has polynomial relation, and may be plotted using a curve. This inference is based upon experiments done by the group on five homeopathic medicines.

The second paper in this issue by Sukul et al studies the intermolecular interaction, $\mathrm{O}-\mathrm{H}$ bonding of Calcarea carb and Silicea in three different UHDs. The authors put forth that UHDs a) possess stronger intermolecular interactions, b) have distinct variation in the number of free water molecules and strength of hydrogen bonding and c) there exists interdrug and inter-potency variations.

The third paper in this issue by Patil et al has brought an important aspect in notice, the standardization of UHD through biological evaluations. Homeopathic medicines have been repeatedly questioned for safety, standardization and quality control checks. But as UHDs are devoid of "active molecules" the standard procedures, unlike for crude starting material, cannot be applied in high dilutions. Patil et al propose to upgrade procedures to include sensitive, reproducible and relevant biological assays to assure quality, efficacy, and safety of homeopathic dilutions.

Homeoprophylaxis (HP) is yet another controversial area on clinical and philosophical grounds, especially use of HP to prevent infectious diseases. To date, we still warrant a systematic study, but none has been performed. Golden and team have taken a leap to conduct two international surveys regarding HP for acceptance, context of use, and preferred remedies, etc., results of which are presented to readers for comments and criticism.

A short communication by Sontag reports the clinically used dosage of homochord Acidum L., as opposed to recommended dosage by the authorities. Although the dose-effect concept is entirely different in homeopathy, we need further research in this area.

Last but not the least, we express our gratitude and acknowledge the valuable contributions of our reviewers, authors and the entire team of IJHDR.

We wish our readers a very happy and prosperous new year 2016.

(C) International Journal of High Dilution Research.

Not for commercial purposes.
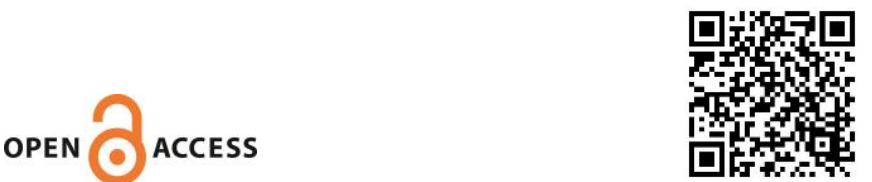

Cite as: Arora S. Potency, Action, Standardization, and Debate - The High Dilution Way [Editorial]. Int J

High Dilution Res. 2015;14(4):1 